\title{
KINEMATICS MODEL AND SIMULATION OF 5-DOF FINGER BASED ON FLEXIBLE PNEUMATIC ACTUATOR
}

\author{
Libin Zhang, Zhiheng Wang, Qinghua Yang, Tiefeng Shao, Guanjun Bao* \\ The MOE Key Laboratory of Mechanical Manufacture and Automation, Zhejiang University \\ of Technology, Hangzhou, Zhejiang, China, 310032 \\ Corresponding author, Address: the MOE Key Laboratory of Mechanical Manufacture and \\ Automation, Zhejiang University of Technology, Hangzhou, Zhejiang, 310032, P. R. China, \\ Tel: +86-571-88320819, Fax: +86-571-88320819,Email: robot@zjut.edu.cn
}

Abstract: $\quad$ Based on flexible pneumatic bending joint and flexible pneumatic spherical joint, a kind of flexible pneumatic 5-DOF finger is proposed, which is composed of two bending joints and a spherical joint. The mathematic model of flexible pneumatic spherical joint is further analyzed. On the researching foundation of flexible pneumatic bending joint and flexible pneumatic spherical joint, the kinematics equation of the 5-DOF finger is deduced. And the redundancy problem of the inverse kinematics solution is resolved by genetic algorithm. The simulation experiment illustrates that genetic algorithm for solving of inverse kinematics is feasible and effective.

Keywords: flexible pneumatic finger, flexible pneumatic actuator, flexible pneumatic bending joint, flexible pneumatic spherical joint

\section{INTRODUCTION}

The complex structure of human hand enables it to grab objects of different size and shape easily, so researching on dexterous hand similar to human hand is many researchers' goal. Many different kinds of multifingered dexterous hand have been developed, such as: the Okada dexterous hand developed by Japanese Electronic Technology Lab in 1974 (Okada,

Zhang, L., Wang, Z., Yang, Q., Shao, T. and Bao, G., 2008, in IFIP International Federation for Information Processing, Volume 259; Computer and Computing Technologies in Agriculture, Vol. 2; Daoliang Li; (Boston: Springer), pp. 777-789. 
1982), the Utah/MIT hand similar to human hand developed by MIT and the University of Utah in 1980 (Mason et al., 1985), the Hitachi hand proposed by Japan in 1984 (Nakano et al., 1984), the DIST hand and UB hand presented by Italy in 1990s (Caffaz et al., 1998), the DLR-1 and DLR-2 Hand developed by German Aerospace Center (Hirzinger et al., 1999; Lovchik et al., 1999), the NASA multi-fingered dexterous hand developed by United States National Aeronautics and NASA Johnson Space Center in 1999, the dexterous hand based on the pneumatic artificial muscles developed by Shadow robot company in England.

Some research institutions in China have also launched the study on dexterous hand in 1980s. The Robot Research Institute of Beijing University of Aeronautics \& Astronautics has developed BH-1, BH-2, BH-3 and BH-4 dexterous hands (Wang et al., 1997; Shang et al., 2000). Harbin University of Technology and German Aerospace Center have jointly developed a new generation of multi-fingered humanoid robot hand HIT/DLR Hand.

The executive components of dexterous hands introduced above are rigid structure, so the adaptability and security for environment and grasp goal are poor and they need more accurate control system. Flexible dexterous hand based on Flexible Pneumatic Actuator FPA has virtues of good flexibility and adaptability, particularly suitable for agriculture fruit picking, service robots and medical rehabilitation apparatuses, which need higher security and adaptability. This paper presents a new kind of 5-DOF finger based on flexible pneumatic bending joint and flexible pneumatic spherical joint. The Kinematics model of this finger is established. The inverse Kinematics equation is also analyzed and simulated. This flexible pneumatic 5-DOF finger can be applied in multi-fingered dexterous hand design, applicable to agricultural harvesting robot, service robots, and other fields.

\section{STRUCTURE AND PRINCIPLE OF FLEXIBLE PNEUMATIC 5-DOF FINGER}

\subsection{Flexible pneumatic actuator FPA}

Yang has proposed the Flexible Pneumatic Actuator FPA, and analyzed its static and dynamic characteristics (Yang et al., 2005). The static model of FPA is:

$$
\Delta L=\frac{\left(P-P_{a t m}\right) R}{2 E_{b} t_{b}-\left(P-P_{a t m}\right) R} L_{b}
$$


where, $\Delta L$ : linear deformation of rubber tube, $t_{b}$ : original thickness of shell, $E_{b}$ : elastic module of FPA, $P_{\text {atm }}$ : atmospheric pressure, $P$ : air pressure in actuator, $R$ : average radius of actuator, $L_{\mathrm{b}}$ : original length of rubber tube.

FPA is the foundation of flexible pneumatic bending joint and flexible pneumatic spherical joint.

\subsection{Flexible pneumatic bending joint}

Based on FPA, Zhang designed the flexible pneumatic bending joint (Zhang et al., 2006). Fig. 1 shows the structure of the bending joint. Adding constraining wire fixed at both ends in one side of FPA rubber tube results in the flexible pneumatic bending joint.
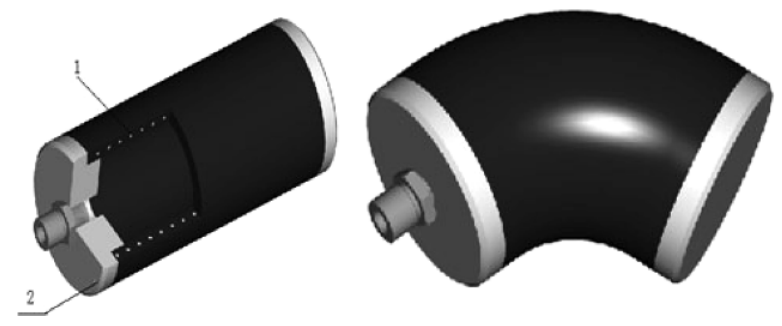

1 Spring; 2 Constraining wire

(a) Original state

(b) Bending state

Fig. 1. Structure of flexible pneumatic bending joint

This kind of joint can realize bending movement in a plane, and the bending angle can be reached at $90^{\circ}$. The static model of the flexible pneumatic bending joint is:

$$
\theta=\frac{L_{b}}{4 r_{b}} \times \frac{\pi r_{b}^{3} \Delta P+6 \pi E_{b} r_{b}^{2} t_{b}-\sqrt{\pi^{2} r_{b}^{6} \Delta P^{2}-20 \pi^{2} \Delta P E_{b}^{2} r_{b}^{5} t_{b}+36 \pi^{2} E_{b}^{2} r_{b}^{4} t_{b}^{2}}}{2 \pi E_{b} r_{b}^{2} t_{b}-\pi r_{b}^{3} \Delta P}
$$

where, $\theta$ : curving angle, $L_{b}$ : original length of rubber tube, $r_{b}$ : average radius of rubber tube, $t_{b}$ : original thickness of shell, $\Delta P$ : the difference between air pressure in the joint and atmospheric pressure, $E_{b}$ : elastic module of the bending joint.

\subsection{Flexible pneumatic spherical joint}

Fig. 2 shows the structure of the flexible pneumatic spherical joint which is composed with three FPAs uniformly distributed by $120^{\circ}$. The working principle of the spherical joint is: if the pressure of compressed air in the three FPAs is adjusted properly, the three FPAs have different elongations. 


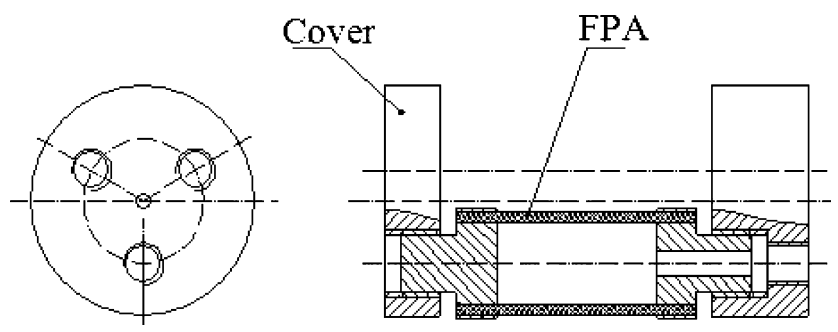

Fig. 2. Structure of flexible pneumatic spherical joint

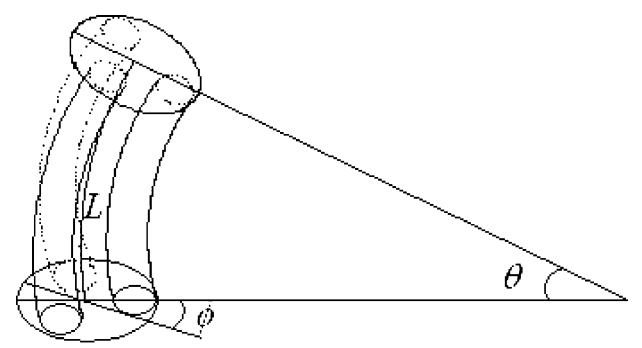

Fig. 3. Bending state of flexible pneumatic spherical joint

The following mathematical model is built:

$$
\left(\begin{array}{l}
P_{1}-P_{a t m} \\
P_{2}-P_{a t m} \\
P_{3}-P_{a t m}
\end{array}\right)=\left(\begin{array}{ccc}
2 & -1-\sqrt{3} t g \phi & -1+\sqrt{3} t g \phi \\
1 & 1 & 1 \\
\cos \phi_{1} & \cos \phi_{2} & \cos \phi_{3}
\end{array}\right)^{-1}\left(\begin{array}{l}
0 \\
\frac{3 E_{b} A_{w}\left(L_{1}-L_{0}\right)}{A_{l} L_{0}} \\
-\frac{E_{b} R A_{w} \theta}{A_{l} L_{0}} \sum_{i=1}^{3} \cos ^{2} \phi_{i}
\end{array}\right)
$$

In practical application, $P_{i}-P_{\mathrm{atm}} \geq 0$, so each matrix item is greater than 0 , when $\phi=0$, the following equation can be obtained:

$$
\begin{aligned}
& \left(\begin{array}{ccc}
2 & -1-\sqrt{3} t g \phi & -1+\sqrt{3} t g \phi \\
1 & 1 & 1 \\
\cos \phi_{1} & \cos \phi_{2} & \cos \phi_{3}
\end{array}\right)\left(\begin{array}{l}
0 \\
\frac{3 E_{b} A_{w}\left(L-L_{0}\right)}{A_{l} L_{0}} \\
-\frac{E_{b} R A_{w} \theta}{A_{l} L_{0}} \sum_{i=1}^{3} \cos ^{2} \phi_{i}
\end{array}\right) \\
& =\frac{E_{b} A_{w}}{4 A_{l} L_{0}}\left[\begin{array}{ccc}
2 & 2 & 0 \\
-1 & 2 & -2 \sqrt{3} \\
-1 & 2 & 2 \sqrt{3}
\end{array}\right]\left(\begin{array}{l}
0 \\
2\left(L_{1}-L_{0}\right) \\
-R \theta
\end{array}\right)
\end{aligned}
$$


From equation (4), the following inequalities can be obtained:

$$
\left\{\begin{array}{l}
L_{1}-L_{0} \geq 0 \\
\left(L_{1}-L_{0}\right)+\frac{\sqrt{3}}{2} R \theta \geq 0, \text { so } L_{1}-L_{0} \geq \frac{\sqrt{3}}{2} R \theta . \text { When the largest spherical } \\
\left(L_{1}-L_{0}\right)-\frac{\sqrt{3}}{2} R \theta \geq 0
\end{array}\right.
$$

joint bending angle is $\theta=\frac{\pi}{2}$, the length of the joint's center line $L_{1}$ can be calculated from the equation:

$$
L_{1}=\frac{\pi \sqrt{3}}{4} R+L_{0}
$$

According to equation (5) and (1), the size of initialization importation air pressure is:

$$
P_{i n}=\frac{\pi E_{b} t_{0} \sqrt{3}}{2 R\left(\frac{\pi \sqrt{3}}{4} R+L_{0}\right)}+P_{a t m}
$$

where, $\phi_{i}$ : angle between shell $i$ and rotation axis $\mathrm{n}, i=1,2,3, \phi$ : abduction angle of the flexible pneumatic finger, $L_{0}$ : original length of the spherical joint's center line, $d$ : distance between the axis of FPA and the axis of the spherical joint, $A_{w}$ : cross-sectional area of FPA shell, $A_{1}$ : cross-sectional area of FPA gas room, $P_{i}$ : air pressure in FPA $i, P_{\mathrm{atm}}$ : atmospheric pressure, $\theta$ : bending angle.

\subsection{Flexible pneumatic 5-DOF finger}

According the anatomical structure of human hand, flexible pneumatic 5-DOF finger is designed based on flexible pneumatic bending joint and flexible pneumatic spherical joint. Fig. 4 shows the structure of the finger.

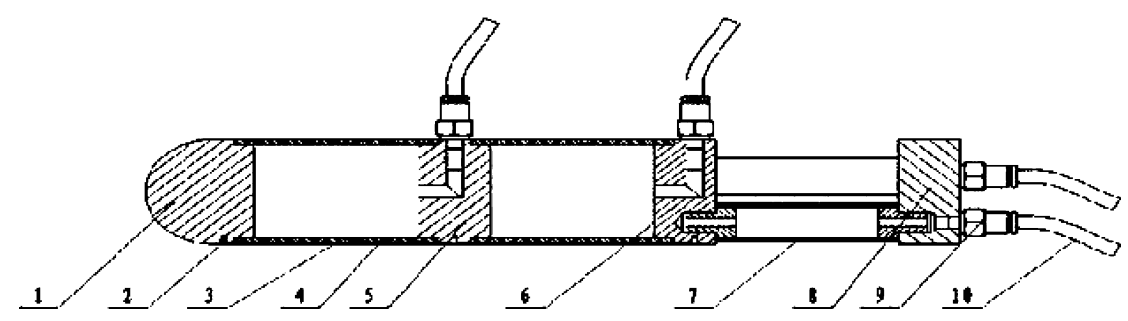

1 Far finger knuckle; 2 Bolt; 3 Constraining wire; 4, 7 FPA;

5 Near finger knuckle; 6 Palm finger knuckle;

8 Carpometacarpal joint; 9 Connector; 10 Air tube

Fig. 4. Structure of flexible pneumatic 5-DOF finger 
Two flexible pneumatic bending joints and one flexible pneumatic spherical joint are connected in series by rigid knuckles. The two constraining wires embedded in the flexible pneumatic bending joints should be maintained in a straight line, and the spherical joint is the first joint near the digital root. The length of the far finger knuckle, near finger knuckle and palm finger knuckle are $l_{3}=15 \mathrm{~mm}, l_{2}=10 \mathrm{~mm}, l_{1}=25 \mathrm{~mm}$ respectively. And the length of the finger joint, metacarpophalangeal joint and carpometacarpal joint are $l_{6}=15 \mathrm{~mm}, l_{5}=30 \mathrm{~mm}, l_{l}=25 \mathrm{~mm}$ respectively.

The flexible pneumatic 5-DOF finger designed in this paper is shown in Fig. 5. Compressed air fills into three joints by five windpipes. When the air pressure inside two bending joints adjusted properly, they can realize different angle bending movement. Controlling the air pressure in three FPAs of the pneumatic spherical joint, the spherical joint can move within $360^{\circ}$ of rotation and bending. When the pressure of air inside the joints gradually reduces, the pneumatic finger reverts to the original state. The flexible pneumatic finger with five DOFs has virtues of excellent flexibility, simple structure, and does not need the complex transmission device and variable speed mechanism. For the use of rubber material as actuators, the finger has good flexibility. When grasping objects, elastics rubber tube can adapt to the shape of the object, and does not cause unnecessary damage to the target.

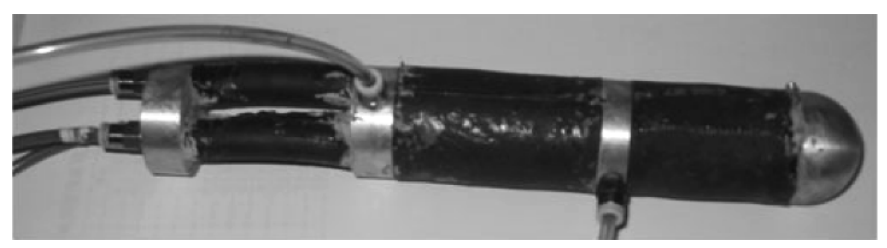

Fig. 5. The photo of the 5-DOF finger

\section{KINEMATICS ANALYSIS OF THE 5-DOF FINGER}

The 5-DOF finger is composed of three joints. Kinematics model of the finger is mainly analyzed for the position and orientation of the fingertip relative to palm space. Fig. 6 shows the coordinate system of the finger. The rotation angle of each joint is $\phi, \theta_{1}, \theta_{2}, \theta_{3}$ respectively.

Coordinate system 0 is fixed on the carpalmetacarpal; its origin $O_{0}$ is the crossing point between axis of the spherical joint and carpalmetacarpal, when the thumb and the other four fingers are in the same plane. The $Z_{0}$-axis is perpendicular to the palm outward, while the $X_{0}$-axis lies along the axis of 

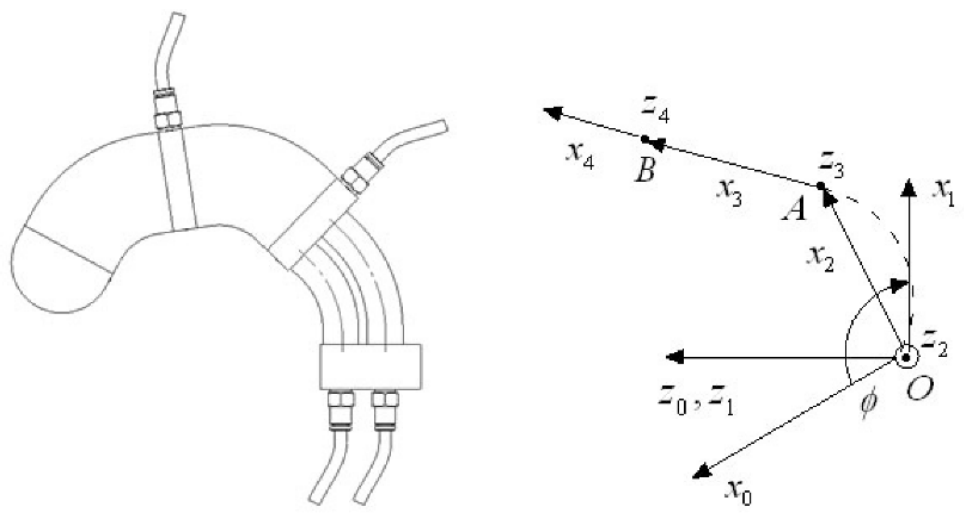

(a) Flexible pneumatic 5-DOF finger (b) Moving analysis of the finger Fig. 6. Coordinate map of flexible pneumatic 5-DOF finger

the joint, and the $Y_{0}$-axis is determined by the right-hand rule. Located in the spherical joint's end connecting with carpalmetacarpal, the coordinate system 1 rotates relative to the coordinate system 0 along with the spherical joint's abduction movement. The origin $O_{1}$ coincides with $O_{0}$, while the $Z_{1}$-axis is perpendicular to the palm outward. Initially, $X_{1}$-axis mergers with $X_{0}$-axis, and then rotates around the $Z_{1}$-axis; while the rotation angle is $\phi$. The $Y_{1}$-axis is determined by the right-hand rule. The origin of the coordinate system 2 coincides with origin $O_{0}$. The $Z_{2}$-axis is perpendicular to bending finger plane, while the $X_{2}$-axis lies along the axis of the joint upward, and the $Y_{2}$-axis is also determined by the right-hand rule. Coordinate systems 3, 4, 5, $6,7,8$ are defined by the method described above. Coordinate 8 is located at the fingertips.

When the abduction angle is $\phi$, Fig.7 shows the simplified model of the finger movement under the coordinate system 1. In Fig. 7, the chord lengths of the three joints 1, 2, 3 are denoted by the symbols $\mathrm{O}_{1} \mathrm{~A}, \mathrm{BC}, \mathrm{DE}$, while the three knuckles' lengths are denoted by the symbols $\mathrm{AB}, \mathrm{CD}, \mathrm{DE}$ respectively. Three symbols $\theta_{1}, \theta_{2}, \theta_{3}$ stand for angles between three knuckles and three chords respectively. According to the geometric relationship, the bending angles of three joints can be calculated, and they are $2 \theta_{1}, 2 \theta_{2}$ and $2 \theta_{3}$. The homogeneous transformation matrixes for the thumb movement are as follows:

$$
T_{0}^{1}=\left[\begin{array}{cccc}
\cos \phi & -\sin \phi & 0 & 0 \\
\sin \phi & \cos \phi & 0 & 0 \\
0 & 0 & 1 & 0 \\
0 & 0 & 0 & 1
\end{array}\right]
$$




$$
\begin{aligned}
& T_{1}^{2}=\left[\begin{array}{cccc}
\cos \theta_{1} & -\sin \theta_{1} & 0 & 0 \\
0 & 0 & -1 & 0 \\
\sin \theta_{1} & \cos \theta_{1} & 0 & 0 \\
0 & 0 & 0 & 1
\end{array}\right] \\
& T_{2}^{3}=\left[\begin{array}{cccc}
1 & 0 & 0 & \frac{l_{4}}{2 \theta_{1}} \sin 2 \theta_{1} \\
0 & 1 & 0 & \frac{l_{4}}{\theta_{1}} \sin ^{2} \theta_{1} \\
0 & 0 & 1 & 0 \\
0 & 0 & 0 & 1
\end{array}\right] \\
& T_{3}^{4}=\left[\begin{array}{cccc}
\cos \theta_{1} & -\sin \theta_{1} & 0 & l_{1} \cos \theta_{1} \\
\sin \theta_{1} & \cos \theta_{1} & 0 & l_{1} \sin \theta_{1} \\
0 & 0 & 1 & 0 \\
0 & 0 & 0 & 1
\end{array}\right] \\
& T_{4}^{5}=\left[\begin{array}{cccc}
\cos \theta_{2} & -\sin \theta_{2} & 0 & \frac{l_{5}}{2 \theta_{2}} \sin 2 \theta_{2} \\
\sin \theta_{2} & \cos \theta_{2} & 0 & \frac{l_{5}}{\theta_{2}} \sin ^{2} \theta_{2} \\
0 & 0 & 1 & 0 \\
0 & 0 & 0 & 1
\end{array}\right] \\
& T_{5}^{6}=\left[\begin{array}{cccc}
\cos \theta_{2} & -\sin \theta_{2} & 0 & l_{2} \cos \theta_{2} \\
\sin \theta_{2} & \cos \theta_{2} & 0 & l_{2} \sin \theta_{2} \\
0 & 0 & 1 & 0 \\
0 & 0 & 0 & 1
\end{array}\right] \\
& T_{6}^{7}=\left[\begin{array}{cccc}
\cos \theta_{3} & -\sin \theta_{3} & 0 & \frac{l_{6}}{2 \theta_{3}} \sin 2 \theta_{3} \\
\sin \theta_{3} & \cos \theta_{3} & 0 & \frac{l_{6}}{\theta_{3}} \sin ^{2} \theta_{3} \\
0 & 0 & 1 & 0 \\
0 & 0 & 0 & 1
\end{array}\right] \\
& T_{7}^{8}=\left[\begin{array}{cccc}
1 & 0 & 0 & l_{3} \cos \theta_{3} \\
0 & 1 & 0 & l_{3} \sin \theta_{3} \\
0 & 0 & 1 & 0 \\
0 & 0 & 0 & 1
\end{array}\right]
\end{aligned}
$$




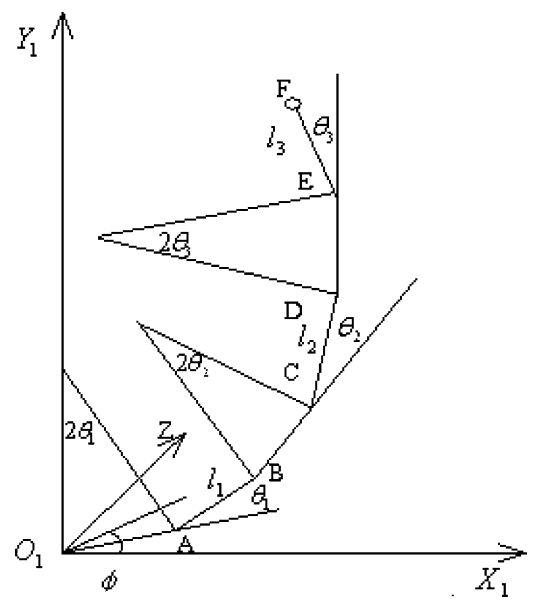

Fig. 7. Simplified model of the finger movement under the coordinate 1

According to the analysis above, we can get the fingertip's position and orientation under the fixed coordinate system 0 :

$$
T_{0}^{8}=T_{0}^{1} T_{1}^{2} T_{2}^{3} T_{3}^{4} T_{4}^{5} T_{5}^{6} T_{6}^{7} T_{7}^{8}
$$

\section{INVERSE KINEMATICS ANALYSIS OF THE 5-DOF FINGER BASED ON GENETIC ALGORITHM}

\subsection{Description of genetic algorithm}

In inverse kinematics analysis, the fingertip positions are given to compute the bending angles of each joint. The bending angles $\phi, \theta_{1}, \theta_{2}$ and $\theta_{3}$ of the three joints can be compute from the equation:

$$
\left[\begin{array}{l}
x \\
y \\
z \\
1
\end{array}\right]=T_{0}^{8} \text {, so we can get the abduction angle: } \phi=\operatorname{actg} \frac{z}{x}, \theta_{1}, \theta_{2} \text { and } \theta_{3}
$$

are determined by the liner equation which has two variable $x_{1}$, and $y_{1}$ in the coordinate system 1 . Where, $x_{1}=\frac{x}{\cos \left(\operatorname{actg} \frac{z}{x}\right)}, y_{1}=y$. According to the 
previous equations, there are an infinite number of solutions for a unique fingertip position. In order to control the finger accurately, we hope the bending angle of each joint has a unique value.

In recent years, along with the development of intelligent control technology, many scholars have used intelligent control solutions to solve the robot inverse kinematics problem. For example, Rasit and Kokera have applied the neural networks to solve the three joints robot's inverse kinematics problem, Hui Shao and Kenzo Nonami have solved the control problem of multi-fingered dexterous hand by using the fuzzy neural network (Shao et al., 2006), and P. Kalra has discussed the application of genetic algorithm for solving the SCARA and PUMA robots' inverse kinematics problem (Kalra et al., 2006).

Genetic algorithm is adopted to solve the inverse kinematics problem of the finger in this paper. The main steps of genetic algorithm are as follows:

(1) Code: The mapping between the genotype and the phenotype is called coding. Binary coding is applied in this paper, and the binary variable median is 20 .

(2) Initial population producing: $N$ data strings are randomly generated, while each data string is called an individual, and then $N$ individuals constitute a group. Initially, genetic algorithm iterates from the $N$ data strings.

(3) Fitness evaluation testing: The fitness function illustrates the individual or solution is superior or interior. The fitness function in this paper is:

$$
\left\{\begin{array}{c}
\min f_{1}=\frac{l_{4}}{2 \theta_{1}} \sin 2 \theta_{1}+l_{1} \cos \theta_{1}+\frac{l_{5}}{2 \theta_{2}} \sin 2 \theta_{2}+l_{2} \cos \theta_{2}+\frac{l_{6}}{2 \theta_{3}} \sin 2 \theta_{3}+l_{3} \cos \theta_{3}-X_{1} f_{1} \geq 0 \\
\min f_{2}=\frac{l_{4}}{\theta_{1}} \sin ^{2} \theta_{1}+l_{1} \sin \theta_{1}+\frac{l_{5}}{\theta_{2}} \sin ^{2} \theta_{2}+l_{2} \sin \theta_{2}+\frac{l_{6}}{\theta_{3}} \sin ^{2} \theta_{3}+l_{3} \sin \theta_{3}-Y_{1} f_{2} \geq 0 \\
0 \leq \theta_{1} \leq \frac{\pi}{3} \quad 0 \leq \theta_{2} \leq \frac{4 \pi}{9} \quad 0 \leq \theta_{3} \leq \frac{\pi}{2}
\end{array}\right.
$$

where, $\left(X_{1}, Y_{1}\right)$ is the target coordinates.

(4) Selection: The individuals in the group which have strong vitality are kept down to the next generation groups by the select operator. According to the fitness value of the individual, genetic algorithm does the operation based on the rule of the survival of the fittest. 
(5) Cross: The group is treated by the crossover operator. Two individuals are selected from the group with the greater probability. The crossover operation exchanges partial parts of the two individuals.

(6) Mutation: Changing one or several bits of the coding string, we obtain the new individuals. The mutation operation can improve the local search ability of genetic algorithm and maintain the genetic diversity of the population.

(7) Stop judgment.

\subsection{Simulation analysis of the inverse kinematics}

Matlab are used to do simulation experiments on genetic algorithm. The number of the individuals is 100 , the number of the maximal genetic generations is 80, and the generation gap is 0.9. Fig. 8 and Fig. 9 show the optimal solutions and tracking performance of the first and the second objective function respectively. From Fig. 8 and Fig. 9, it can be seen that the 10th generation function solutions tend to be 0 , which are the expected values. So the No.15 generation group can be the inverse kinematics solutions of $\theta_{1}, \theta_{2}$, and $\theta_{3}$. Fig. 10 shows the optimal solutions tracking of the two objective functions.

Through the simulation, the redundancy problem on the inverse kinematics of the 5-DOF finger can be solved by the genetic algorithm. The method is simple and clear, no redundant constraints.

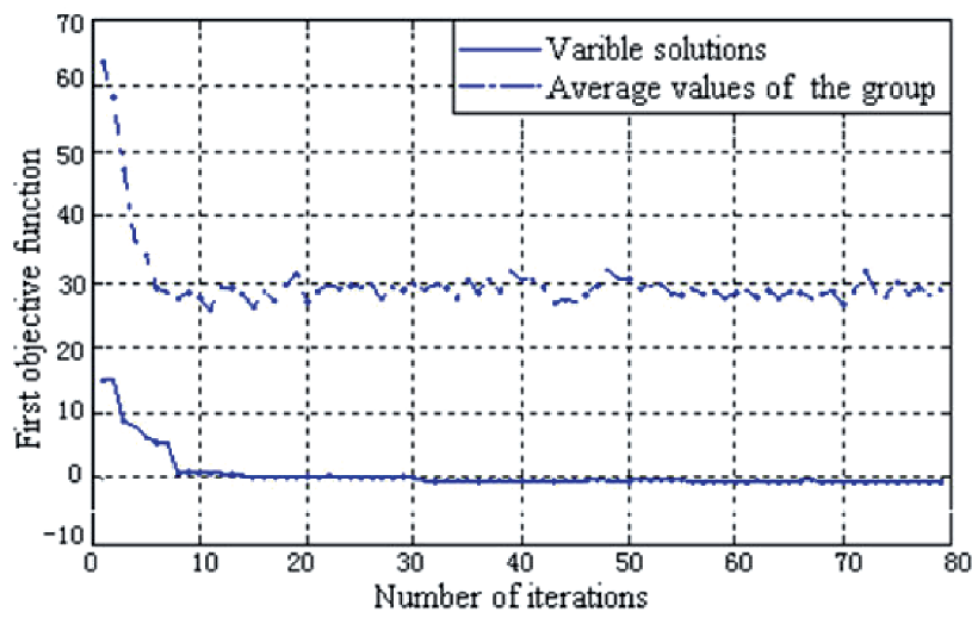

Fig. 8. Optimal solutions and tracking performance of the first 


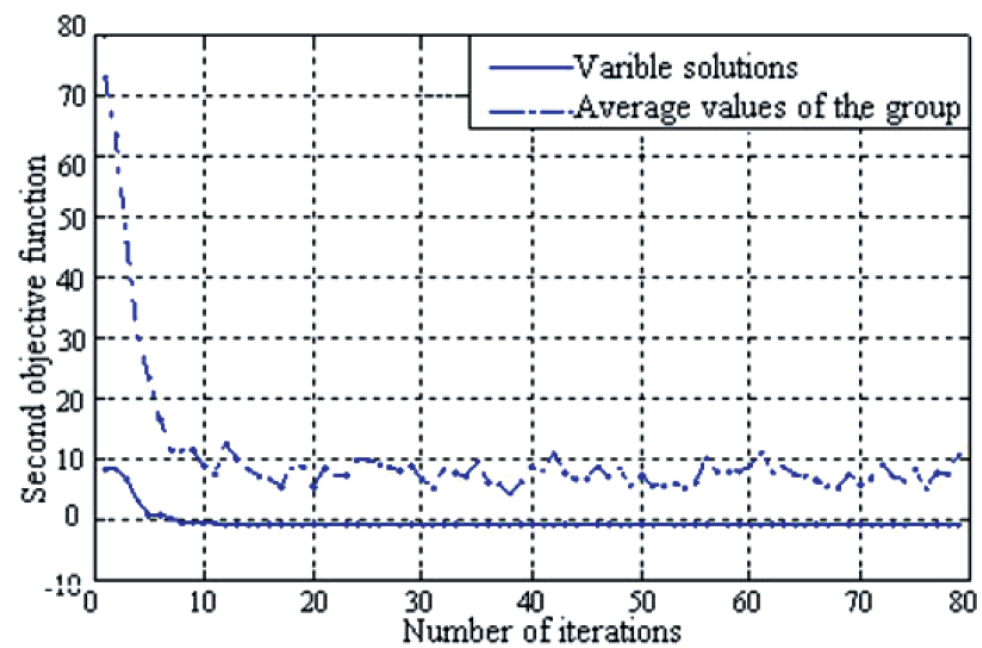

Fig. 9. Optimal solutions and tracking performance of the second objective function after 80 iterations

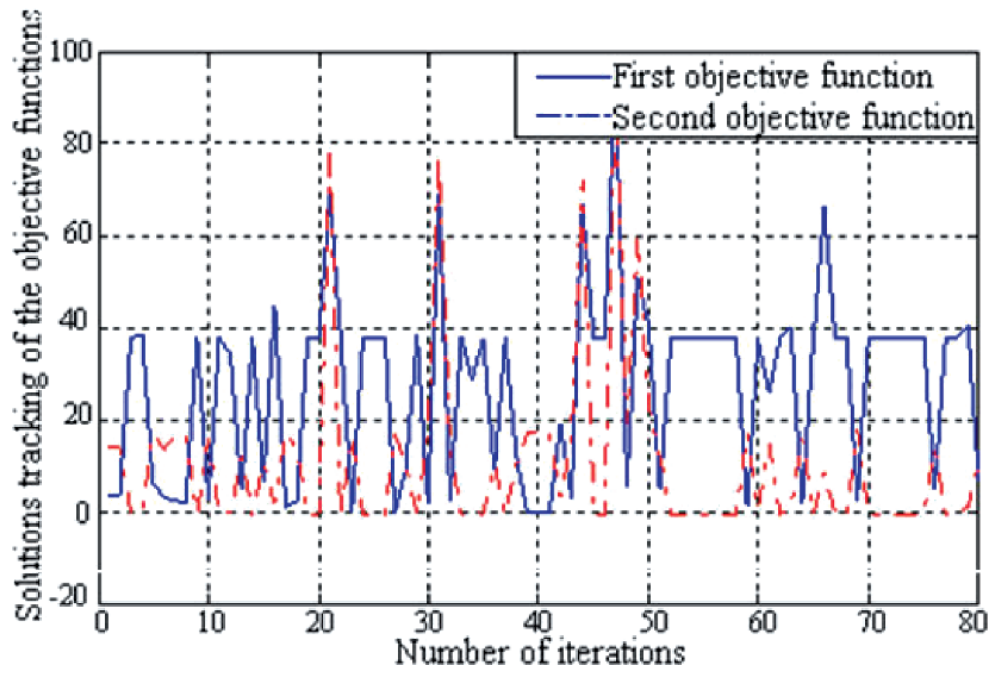

Fig. 10. Optimal solutions tracking of the two objective functions after 80 iterations 


\section{CONCLUSION}

(1) Based on the flexible pneumatic bending joint and flexible pneumatic spherical joint, flexible pneumatic 5-DOF finger is proposed. It has many advantages such as good flexibility, simple structure and good adaptability.

(2) Kinematics characteristic of the 5-DOF finger is profoundly studied and the kinematics model is built.

(3) Inverse kinematics characteristic of the flexible pneumatic 5-DOF finger is analyzed, and the genetic algorithm is applied to solve the inverse kinematics equation. The simulation experiments show that applying genetic algorithm to solve the inverse kinematics problem is feasible and effective.

\section{REFERENCES}

Caffaz, Cannata G. The Design and Develoopment of the DIST-Hand Dextrous Gripper [J]. Proceedings of the IEEE International Conference on Robotics and Automation, Leuven, Belgium. 1998:2075-2080.

Hirzinger G., Fischer M., Brunne B. Advances in Robotics: The DLR Experience [J]. The International Journal of Robotics Research, 1999, 18(1): 1064-1087.

http://robonaut.jsc.nasa.gov/robonaut.html

http://www.dlr.de/rm/en/desktopdefault.aspx/tabid-117/

http://www.hitrobot.net/

http://www.shadow.org.uk/projects/openhardware.shtml

http://wwwrobot.gmc.ulaval.ca/liens/liens_a.html

Hui Shao, Kenzo Nonamib, Tytus Wojtara, etc. Neuro-fuzzy position control of demining tele-operation system based on RNN modeling [J]. Robotics and Computer-Integrated Manufacturing, 2006, 22: 25-32.

Libin Zhang, Guanjun Bao, Qinghua Yang, Jian Ruan and Liyong Qi. Static Model of Flexible Pneumatic Bending Joint, Proceeding of the 2006 9th Int. Conf. Control, Automation, Robotics and Vision, Singapore, 2006.12: 1749-1753.

Lovchik C. S., MDifler M. A. The Robonaut Hand: A Dextrous Robotic Hand for Space [J]. Proceedings for the IEEE International Conference on Robotics and Automation. Detroit, Michigan, 1999: 907-912.

Mason M. T., Salisbury J. K. Robot Hands and the Mechanics of Manipulation [J]. MIT Press, Cambridge, USA, 1985: 3-93.

Nakano Y., Fujie M., Hosada Y. Hitachi's Robot Hand [J]. Robotics Age, 1984, 6(7): 18-20.

Okada T. Computer control of multi-jointed finger system for precise object-handing [J]. IEEE Transactions on Systems, Man and Cybernetics, 1982, 12(3): 289-299.

P. Kalra, P.B. Mahapatra, D.K. Aggarwal. An evolutionary approach for solving the multimodal inverse kinematics problem of industrial robots [J]. Mechanism and Machine Theory, 2006, 41: 1213-1229. 
Rasit Koker, CemilOza, Tarık, etc. A study of neural network based inverse kinematics solution for a three-joint robot [J], Robotics and Autonomous Systems 2004, 49: 227-234.

Shang Xisheng, Guo Weidong, Zhan Hao, etc, Grasp Planning and Realization of BH-4 Dexterous Hand [J], Robot, 2000, 22(7): 608-612 (in Chinese).

Wang Guoqing, Zhang Qixian, Li Dazhai, He Yongqiang, Grasping Control of the Dexterous Hand Based on the Degree of Stability of Grasping [J]. Acta Aeronautica Et Astronautica Sinica, 1997, 18(3): 294-298 (in Chinese).

Yang Qinghua, Research on Flexible Pneumatic Joints and Their Application Based on Flexible Pneumatic Actuator, Dissertation for Doctor's Degree in Engineering of Zhangjiang University of technology, 2005 (in Chinese).

Yang Qinghua, Zhang Libin, Bao Guanjun, Ruan Jian, Pneumatic Squirming Robot Based on Flexible Pneumatic Actuator, Proceedings of SPIE ICMIT2005: Control Systems and Robotics, Sept. 2005, Vol. 6042: 60422w-1-60422w-5.

Zhang Peiyan, Lv Tiansheng, Song Libo, Study on BP Networks-based Inverse Kinematics of Motoman Manipulator [J], Mechanical \& Electrical Engineering Magazine, 2004, 20(2): 56-58 (in Chinese). 